\title{
SMALL SOLUTIONS OF QUADRATIC CONGRUENCES
}

\author{
by D. R. HEATH-BROWN
}

To Robert Rankin on the occasion of his 70 th birthday

1. Introduction. Let $Q(\mathbf{x})=Q\left(x_{1}, \ldots, x_{n}\right) \in \mathbb{Z}\left[x_{1}, \ldots, x_{n}\right]$ be a quadratic form. We investigate the size of the smallest non-zero solution of the congruence $Q(\mathbf{x}) \equiv 0(\bmod q)$. We seek a bound $B_{n}(q)$, independent of $Q$, such that there is always a non-zero solution satisfying

$$
\operatorname{Max}_{1 \leqslant i \leqslant n}\left|x_{i}\right| \leqslant B_{n}(q)
$$

The form $Q(x)=\sum_{i}^{n} x_{i}^{2}$ gives the trivial lower bound $B_{n}(q) \geqslant(q / n)^{1 / 2}$ for all $q$ and $n$, since if $\mathbf{x} \neq \mathbf{0}$ and $q \mid Q(\mathbf{x})$, then $Q(\mathbf{x}) \geqslant q$.

It was shown by Schinzel, Schlickewei and Schmidt [3] that

$$
B_{n}(q) \leqslant q^{1 / 2+1 /(4(n-1) / 21+2)}, \quad(n \geqslant 3) .
$$

They used this to obtain Diophantine approximation results for $\|Q(\mathbf{x})\|$, in which $Q$ is a quadratic form with real coefficients. It is reasonable to conjecture that

$$
B_{n}(q) \ll q^{1 / 2+\varepsilon},
$$

for any $\varepsilon>0$, as soon as $n \geqslant 4$, but no general improvement on (1) is known. However we shall show that the above conjecture is indeed true if $q$ is restricted to prime values.

THEOREM 1. We have $B_{n}(p) \ll p^{1 / 2}(\log p)$ uniformly for $n \geqslant 4$, where $p$ is prime.

Indeed using the method of $[3]$ we shall easily prove a stronger result in certain cases.

THEOREM 2. Let $p$ be an odd prime and take $n=4$. If $p \mid \operatorname{det} Q$ or $\left(\frac{\operatorname{det} Q}{p}\right)=1$ then $p \mid Q(\mathbf{x})$ for some $\mathbf{x} \in \mathbb{Z}^{4}-\{0\}$, with $\operatorname{Max}\left|x_{i}\right| \leqslant p^{1 / 2}$.

Here $\operatorname{det} Q$ is the determinant of the integer matrix representing $Q$, and $\left(\frac{\dot{p}}{p}\right)$ is the Legendre symbol.

The condition $n \geqslant 4$ in Theorem 1 , and in the general conjecture (2), is in fact necessary. Indeed if $n=3$ the bound (1) is essentially best possible, even when $q$ is restricted to be prime.

THEOREM 3. For all primes $p$ we have $B_{3}(p) \geqslant p^{2 / 3}+O\left(p^{1 / 3}\right)$.

The forms used in proving Theorem 3 are all singular $(\bmod p)$. It is reasonable to conjecture that $B_{3}^{*}(p) \ll p^{1 / 2+\varepsilon}$, where $B_{n}^{*}(p)$ is defined analogously to $B_{n}(p)$, but with the forms $Q$ restricted to be non-singular $(\bmod p)$.

In what follows $\mathbf{x}, \mathbf{y}$, etc. will always be column vectors in $\mathbb{R}^{4}$ or $\mathbb{Z}^{4}$ as appropriate. We

Glasgow Math. J. 27 (1985) 87-93. 
denote the zero vector by $\mathbf{0}$. We write $\mathbf{x} \cdot \mathbf{y}$ for the usual scalar product $\mathbf{x}^{T} \mathbf{y}$. By " $\left|x_{i}\right| \leqslant B$ " we shall mean that $\left|x_{i}\right| \leqslant B$ for $1 \leqslant i \leqslant 4$. We will write $\mathbf{x}(\bmod p)$, as a summation condition, to mean that each component $x_{i}$ runs from 1 to $p$. If $p \nmid k$ we write $\bar{k}$ for the inverse of $k(\bmod p)$. The quadratic form $Q$ will also be thought of as a matrix, also denoted by $Q$, with entries in the field of $p$ elements. (We will always take $p \geqslant 3$.) With this convention $Q^{-1}$ will be another quadratic form, with coefficients defined $(\bmod p)$.

2. The Proof of Theorem 3. We shall prove the theorems in reverse order, starting with Theorem 3. Let $a$ be a quadratic non-residue of $p$ and let $b=\left[p^{1 / 3}\right]$. We take

$$
Q=\left(x_{1}-b x_{2}\right)^{2}-a\left(x_{2}-b x_{3}\right)^{2} \text {. }
$$

Then if $p \mid Q$ we must have $x_{1} \equiv b x_{2}(\bmod p)$ and $x_{2} \equiv b x_{3}(\bmod p)$. Now if $x_{1} \neq b x_{2}$ we have $\left|x_{1}-b x_{2}\right| \geqslant p$, whence

Similarly, if $x_{2} \neq b x_{3}$ then

$$
(1+b) \operatorname{Max}\left(\left|x_{1}\right|,\left|x_{2}\right|\right) \geqslant p \text {. }
$$

It follows that

$$
(1+b) \operatorname{Max}\left(\left|x_{2}\right|,\left|x_{3}\right|\right) \geqslant p .
$$

$$
\operatorname{Max}_{1 \leqslant i \leqslant 3}\left|x_{i}\right| \geqslant(1+b)^{-1} p=p^{2 / 3}+O\left(p^{1 / 3}\right),
$$

unless $x_{1}=b x_{2}$ and $x_{2}=b x_{3}$. In the latter case a non-zero solution must have $x_{3} \neq 0$, whence

$$
\operatorname{Max}_{1 \leqslant i \leqslant 3}\left|x_{i}\right| \geqslant\left|x_{1}\right|=b^{2}\left|x_{3}\right| \geqslant b^{2}=p^{2 / 3}+O\left(p^{1 / 3}\right) \text {. }
$$

This completes the proof of Theorem 3.

3. The Proof of Theorem 2. We begin by showing that, under the conditions of Theorem 2, there are two linear forms $L_{1}(\mathbf{x}), L_{2}(\mathbf{x})$ such that $p \mid Q(\mathbf{x})$ whenever $L_{1}(\mathbf{x}) \equiv$ $L_{2}(\mathbf{x}) \equiv 0(\bmod p)$. To do this we work in the field $\mathbb{F}_{\mathrm{p}}$ of $p$ elements, and look for a form $Q^{\prime}\left(x_{1}^{\prime}, \ldots, x_{4}^{\prime}\right)$, equivalent to $Q$, such that $Q^{\prime}=0$ when $x_{1}^{\prime}=x_{2}^{\prime}=0$. If $Q$ has rank 2 or less this is immediate, since $Q$ is equivalent to a form $Q^{\prime}\left(x_{1}^{\prime}, x_{2}^{\prime}\right)$. If $Q$ has rank 3 , then it can be transformed into $Q^{\prime}\left(x_{1}^{\prime}, x_{2}^{\prime}, x_{3}^{\prime}\right)$. By Chevalley's Theorem the latter is a zero form and so is equivalent to $Q^{\prime \prime}\left(x_{1}^{\prime \prime}, x_{2}^{\prime \prime}, x_{3}^{\prime \prime}\right)$ with $Q^{\prime \prime}(0,0,1)=0$. Hence $Q^{\prime \prime}=0$ if $x_{1}^{\prime \prime}=x_{2}^{\prime \prime}=0$. Finally, if $Q$ is non-singular then it is equivalent (see for example Borevich and Shafarevich [1, Theorem 7, p. 394]) to $Q^{\prime}=2 x_{1}^{\prime} x_{2}^{\prime}+Q_{0}\left(x_{3}^{\prime}, x_{4}^{\prime}\right)$, since $Q$ is a zero form by Chevalley's Theorem. Here $\operatorname{det} Q_{0}=-\operatorname{det} Q$, so that $-\operatorname{det} Q_{0}$ is a square in $\mathbb{F}_{\mathrm{p}}$. Thus $Q_{0}$ factorizes as $Q_{0}=2 x_{5}^{\prime} x_{6}^{\prime}$, whence $Q^{\prime}=0$ for $x_{1}^{\prime}=x_{5}^{\prime}=0$. The existence of $L_{1}, L_{2}$ now follows in all cases.

The conditions $L_{1}(\mathbf{x}) \equiv L_{2}(\mathbf{x}) \equiv 0(\bmod p)$ define a sublattice of $\mathbb{Z}^{4}$ of determinant $p^{2}$. It follows from Minkowski's linear forms theorem that there is some non-zero point on the lattice with $\operatorname{Max}\left|x_{i}\right| \leqslant p^{1 / 2}$, and Theorem 2 is proved. (See for example Hardy and Wright [2; Theorem 448]. To apply the theorem as it is stated there we note that there is a $4 \times 4$ matrix $M$, of determinant $p^{2}$, such that $\xi$ is in the above lattice if and only if $\xi=M \mathbf{x}$ for some $\mathbf{x} \in \mathbb{Z}^{4}$.) 
4. Proof of Theorem 1; preliminaries. We observe at the outset that it suffices to consider the case $n=4$, since in general one may examine the quaternary form obtained from $Q$ by setting $x_{5}=\ldots=x_{n}=0$. Moreover, by Theorem 2 , we may suppose that $\left(\frac{\operatorname{det} Q}{p}\right)=-1$. Finally, we may take $p \geqslant 3$.

Our key tool is the Poisson summation formula applied to suitable functions $f: \mathbb{R}^{4} \rightarrow \mathbb{R}$. These will have Fourier transform

$$
\hat{f}(y)=\int_{\mathbb{R}^{4}} f(\mathbf{x}) e(-\mathbf{x} \cdot \mathbf{y}) d x_{1} \ldots d x_{4} .
$$

Here we have set $e(u)=\exp (2 \pi i u)$; we shall also use $e_{\mathrm{p}}(u)$, defined to be $e(u / p)$.

LEMMA 1. We have

where

$$
\sum_{\mathbf{x} \in \mathbb{Z}^{4}, p \mid Q(\mathbf{x})} f(\mathbf{x})=p^{-5} \sum_{\mathbf{y} \in \mathbb{Z}^{4}} S_{p}(\mathbf{y}) \hat{f}\left(\frac{1}{p} \mathbf{y}\right)
$$

$$
S_{\mathrm{p}}(\mathbf{y})=\sum_{s=1}^{p} \sum_{t(\bmod p)} e_{p}(s Q(\mathbf{t})+\mathbf{y} \cdot \mathbf{t}) .
$$

Proof. The left hand side of (3) is

$$
\frac{1}{p} \sum_{s=1}^{p} \sum_{\mathbf{x} \in \mathbb{Z}^{4}} e_{p}(s Q(\mathbf{x})) f(\mathbf{x})=\frac{1}{p} \sum_{s=1}^{p} \sum_{\mathbf{t}(\bmod p)} e_{p}(s Q(\mathbf{t})) \sum_{\mathbf{u} \in \mathbb{Z}^{4}} f(\mathbf{t}+p \mathbf{u}) .
$$

We apply the Poisson summation formula to $g(\mathbf{u})=f(\mathbf{t}+p \mathbf{u})$. This gives

$$
\sum_{\mathbf{u} \in \mathbb{Z}^{4}} g(\mathbf{u})=\sum_{\mathbf{y} \in \mathbb{Z}^{4}} \hat{g}(\mathbf{y})
$$

and since

$$
\hat{g}(\mathbf{y})=p^{-4} e_{p}(\mathbf{y} \cdot \mathbf{t}) \hat{f}\left(\frac{1}{p} \mathbf{y}\right)
$$

Lemma 1 follows.

LEMMA 2. Let $\left(\frac{\operatorname{det} Q}{p}\right)=-1$. Then

where

$$
S_{\mathbf{p}}(\mathbf{y})=p^{2}+p^{4} Y(\mathbf{y})-p^{3} Z(\mathbf{y})
$$

$$
Y(\mathbf{y})=\left\{\begin{array}{ll}
1, & p \mid \mathbf{y}, \\
0, & p \nmid \mathbf{y},
\end{array} \quad Z(\mathbf{y})= \begin{cases}1, & p \mid Q^{-1}(\mathbf{y}) \\
0, & p \nmid Q^{-1}(\mathbf{y})\end{cases}\right.
$$

Proof. We begin by diagonalizing $Q$. Choose $R$, invertible $(\bmod p)$, such that $Q=$ $R^{\mathrm{T}} D R$, with $D=\operatorname{Diag}\left(d_{1}, \ldots, d_{4}\right)$. We substitute $R \mathbf{t}=\mathbf{u}$ in (4), whence $Q(\mathbf{t})=D(\mathbf{u})$ and

$$
\mathbf{y} \cdot \mathbf{t}=\mathbf{y}^{T} \mathbf{t}=\mathbf{y}^{T} R^{-1} \mathbf{u}=\mathbf{v}^{T} \mathbf{u}=\mathbf{v} \cdot \mathbf{u}
$$


with

$$
\mathbf{v}=\left(R^{-1}\right)^{\mathrm{T}} \mathbf{y}
$$

Thus

$$
\begin{aligned}
S_{\mathbf{p}}(\mathbf{y}) & =\sum_{s=1}^{p} \sum_{\mathbf{u}(\bmod \mathbf{p})} e_{\mathrm{p}}(s D(\mathbf{u})+\mathbf{v} \cdot \mathbf{u}) \\
& =p^{4} Y(\mathbf{v})+\sum_{s=1}^{p-1} \prod_{i=1}^{4}\left\{\sum_{u_{i}=1}^{p} e_{\mathrm{p}}\left(s d_{i} u_{i}^{2}+v_{i} u_{i}\right)\right\} .
\end{aligned}
$$

Here the term $Y(\mathbf{v})$ is the contribution from $s=p$. From (5) we have $Y(\mathbf{v})=Y(\mathbf{y})$. Each of the innermost sums in (6) is a standard Gauss sum of the form

$$
\sum_{u=1}^{p} e_{p}\left(a u^{2}+b u\right)=\tau_{p}\left(\frac{a}{p}\right) e_{p}\left(-\overline{4 a} b^{2}\right), \quad(p \nmid 4 a) .
$$

Moreover $\tau_{p}^{4}=p^{2}$ and

$$
\prod_{i=1}^{4}\left(\frac{s d_{i}}{p}\right)=\left(\frac{\operatorname{det} D}{p}\right)=\left(\frac{\operatorname{det} Q}{p}\right)=-1
$$

Thus (6) becomes

$$
p^{4} Y(\mathbf{y})-p^{2} \sum_{s=1}^{p-1} e_{p}\left(-\overline{4 s} D^{-1}(\mathbf{v})\right)
$$

Finally we observe that

$$
\sum_{s=1}^{\mathrm{p}-1} e_{\mathrm{p}}(-\overline{4 s} k)=\sum_{\mathrm{t}=1}^{\mathrm{p}-1} e_{\mathrm{p}}(t k)=\left\{\begin{array}{rr}
p-1, & p \mid k \\
-1, & \mathrm{p} \nless k
\end{array}\right.
$$

and that

$$
D^{-1}(\mathbf{v})=\mathbf{v}^{\mathrm{T}} D^{-1} \mathbf{v}=\mathbf{y}^{\mathrm{T}} Q^{-1} \mathbf{y}=Q^{-1}(\mathbf{y})
$$

Lemma 2 now follows.

Lemmas 1 and 2 now yield

$$
\sum_{\mathbf{x} \in \mathbb{Z}^{4}, p \mid Q(\mathbf{x})} f(\mathbf{x})=p^{-3} \sum_{\mathbf{y} \in \mathbb{Z}^{4}} \hat{f}\left(\frac{1}{p} \mathbf{y}\right)+p^{-1} \sum_{\mathbf{y} \in \mathbb{Z}^{4}} \hat{f}(\mathbf{y})-p^{-2} \sum_{\mathbf{y} \in \mathbf{y}^{4}, p \mid \mathbf{Q}^{-1}(\mathbf{y})} \hat{f}\left(\frac{1}{p} \mathbf{y}\right) .
$$

We may apply the Poisson summation formula again to the first two sums on the right to produce the following result.

LEMMA 3. We have

$$
\sum_{\mathbf{x} \in \mathbb{Z}^{4}, p \mid Q(\mathbf{x})} f(\mathbf{x})=p^{-1} \sum_{\mathbf{x} \in \mathbb{Z}^{4}} f(\mathbf{x})+p \sum_{\mathbf{x} \in \mathbb{Z}^{4}} f(p \mathbf{x})-p^{-2} \sum_{\mathbf{y} \in \mathbb{Z}^{4}, p \mid \mathbb{Q}^{-1}(\mathbf{y})} \hat{f}\left(\frac{1}{p} \mathbf{y}\right) .
$$

Our choice of $f$ will be based on the function considered overleaf. 
LEMMA 4. Define $g(x)=\left\{\begin{aligned} 1-|x|, & |x| \leqslant 1, \\ 0, & |x| \geqslant 1,\end{aligned}\right.$ so that $\hat{g}(y)=\left(\frac{\sin \pi y}{\pi y}\right)^{2}$. Let $h(x)=(g * g * g)(x)$. Then

(i) Supp $h \subseteq[-3,3]$,

(ii) $0 \leqslant h(x) \leqslant 1$ for all $x$,

(iii) $h(x) \geqslant \frac{1}{32}$ for $|x| \leqslant \frac{1}{4}$,

(iv) $\hat{h}(y)=\left(\frac{\sin \pi y}{\pi y}\right)^{6}$.

Proof. We have

$$
h(x)=\int_{-\infty}^{\infty} \int_{-\infty}^{\infty} g(u) g(v-u) g(x-v) d u d v .
$$

Thus, if $h(x) \neq 0$, there must exist $u$, v such that $|u| \leqslant 1,|v-u| \leqslant 1$, and $|x-v| \leqslant 1$. This requires $|x| \leqslant 3$, proving part (i). The lower bound $h \geqslant 0$ is immediate from (7). Moreover

$$
\begin{aligned}
h(x) & \leqslant \int_{-\infty}^{\infty} \int_{-\infty}^{\infty} g(u) g(v-u) d u d v \\
& =\int_{-\infty}^{\infty} \int_{-\infty}^{\infty} g(u) g(w) d u d w \\
& =\hat{g}(0)^{2}=1,
\end{aligned}
$$

which establishes part (ii). For part (iii) we note that if $|u|,|v|,|x| \leqslant \frac{1}{4}$, then $g(u), g(v-u), g(x-v) \geqslant \frac{1}{2}$, while the corresponding area of integration in $(7)$ is $\left(\frac{1}{2}\right)^{2}$. Finally (iv) follows from the convolution formula for fourier integrals.

5. Proof of Theorem 1. We begin by applying Lemma 3 with the function

$$
f(\mathbf{x})=f_{D}(\mathbf{x})=\prod_{i=1}^{4} h\left(x_{i} / D\right) \text {. }
$$

From Lemma 4 parts (i) and (ii) we have

$$
\begin{aligned}
\sum f_{D}(\mathbf{x}) & \leqslant \#\left\{\mathbf{x} \in \mathbb{Z}^{4} ;\left|x_{i}\right| \leqslant 3 D\right\} \ll D^{4}, \\
\sum f_{D}(p \mathbf{x}) & \leqslant \#\left\{\mathbf{x} \in \mathbb{Z}^{4} ;\left|x_{i}\right| \leqslant 3 D / p\right\}=1, \quad(D<p / 3) .
\end{aligned}
$$

By Lemma 4 part (iii) we have $f_{D}(\mathbf{x}) \gg 1$ for $\left|x_{i}\right| \leqslant D / 4$, and by part (iv) we have $\hat{f}_{D}(\mathbf{y}) \geqslant 0$. We deduce the following result.

LEMMA 5. If $\left(\frac{\operatorname{det} Q}{p}\right)=-1$ and $D<p / 3$ then

$$
\#\left\{\mathbf{x} ;\left|x_{i}\right| \leqslant D / 4, p \mid Q(\mathbf{x})\right\} \ll p^{-1} D^{4}+p .
$$

Since $Q(x) \equiv 0(\bmod p)$ has $O\left(p^{3}\right)$ solutions $(\bmod p)$, the lemma is clearly true for $D \geqslant p / 3$ too. 
We can improve Lemma 5 for small values of $D$. Suppose that $D \leqslant \frac{1}{4} p^{1 / 2}$ and put $P=p^{1 / 2}(2 D)^{-1}$. Consider primes $q$ in the range $P<q \leqslant 2 P$. If $p \mid Q(\mathbf{x})$ with $\left|x_{i}\right| \leqslant D$, then $p \mid Q(q \mathbf{x})$ and $\left|q x_{i}\right| \leqslant p^{1 / 2}$. Hence

$$
(\pi(2 P)-\pi(P)) \#\left\{\mathbf{x} \neq \mathbf{0} ;\left|x_{i}\right| \leqslant D, p \mid Q(\mathbf{x})\right\} \leqslant \sum_{\mathbf{y}} \#\{q ; P<q \leqslant 2 P, q \mid \mathbf{y}\}
$$

where $\mathbf{y} \in \mathbb{Z}^{\mathbf{4}}-\{\mathbf{0}\}$ satisfies $\left|y_{i}\right| \leqslant p^{1 / 2}, p \mid Q(\mathbf{y})$. However, if $\mathbf{y} \neq \mathbf{0}$ then

$$
\#\{q ; P<q \leqslant 2 P, q \mid \mathbf{y}\} \leqslant \frac{\log p^{1 / 2}}{\log P} .
$$

Moreover $\pi(2 P)-\pi(P) \gg \frac{P}{\log P}$, whence

$$
\begin{aligned}
\#\left\{\mathbf{x} \neq \mathbf{0} ;\left|x_{i}\right| \leqslant D, p \mid Q(\mathbf{x})\right\} & \ll P^{-1}(\log p) . \#\left\{\mathbf{y} ;\left|y_{i}\right| \leqslant p^{1 / 2}, p \mid Q(\mathbf{y})\right\} \\
& \ll P^{-1} p(\log p),
\end{aligned}
$$

by Lemma 5 . On using Lemma 5 itself for $D \geqslant \frac{1}{4} p^{1 / 2}$ we now have the following result.

LEMMA 6. If $\left(\frac{\operatorname{det} Q}{p}\right)=-1$ then

$$
\#\left\{\mathbf{x} \in \mathbb{Z}^{4} ;\left|x_{i}\right| \leqslant D, p \mid Q(\mathbf{x})\right\} \ll D^{4} p^{-1}+D p^{1 / 2}(\log p) .
$$

We apply this not to $Q$ but to $Q^{-1}$, noting that $\left(\frac{\operatorname{det} Q^{-1}}{p}\right)=\left(\frac{\operatorname{det} Q}{p}\right)=-1$. We take $f=f_{B}$, with $p^{1 / 2}<B<p$, in Lemma 3 , whence

$$
\begin{aligned}
\hat{f}_{B}\left(\frac{1}{p} \mathbf{y}\right)=B^{4} \prod_{i=1}^{4} & \left(\frac{\sin \pi y_{i} B / p}{\pi y_{i} B / p}\right)^{6} \ll B^{4} \prod_{i=1}^{4} \operatorname{Min}\left(1,\left(\frac{p}{B\left|y_{i}\right|}\right)^{6}\right) \\
& \ll B^{4} \operatorname{Min}\left(1,\left(\frac{p / B}{\operatorname{Max}\left|y_{i}\right|}\right)^{6}\right) .
\end{aligned}
$$

We proceed to bound

$$
\sum_{\mathbf{y} \in \mathbb{Z}^{4}, \mathbf{p} \mid Q^{-1}(\mathbf{y})} \hat{f}\left(\frac{1}{p} \mathbf{y}\right)
$$

The term $\mathbf{y}=\mathbf{0}$ contributes $O\left(B^{4}\right)$. We group the remaining terms into ranges $\frac{1}{2} D<$ $\operatorname{Max}\left|y_{i}\right| \leqslant D$, where $D$ is a power of 2 . In such a range there are, by Lemma 6 , $\ll D^{4} p^{-1}+D p^{1 / 2}(\log p)$ terms, and each is of magnitude $\ll B^{4} \operatorname{Min}\left(1,\left(\frac{p}{B D}\right)^{6}\right)$. The total
for $D \leqslant p / B$ is thus

$$
\begin{aligned}
\ll B^{4} \sum_{\mathrm{D}}\left(D^{4} p^{-1}+D p^{1 / 2}(\log p)\right) & \ll B^{4}\left(\left(\frac{p}{B}\right)^{4} p^{-1}+\left(\frac{p}{B}\right) p^{1 / 2}(\log p)\right) \\
& \ll p^{3}+p^{3 / 2} B^{3}(\log p),
\end{aligned}
$$


while for $D \geqslant p / B$ it is

$$
\begin{aligned}
& \ll B^{-2} p^{6} \sum_{D}\left(D^{-2} p^{-1}+D^{-5} p^{1 / 2}(\log p)\right) \\
& \ll B^{-2} p^{6}\left(\left(\frac{p}{B}\right)^{-2} p^{-1}+\left(\frac{p}{B}\right)^{-5} p^{1 / 2}(\log p)\right) \\
& \ll p^{3}+p^{3 / 2} B^{3}(\log p) .
\end{aligned}
$$

Hence

$$
p^{-2} \sum_{\mathbf{y} \in \mathbb{Z}^{2}, \mathbf{p} \mid \mathbf{Q}^{-1}(\mathbf{y})} \hat{f}_{\mathbf{B}}\left(\frac{1}{p} \mathbf{y}\right) \ll p^{-2} B^{4}+p+p^{-1 / 2} B^{3}(\log p) \ll p^{-1 / 2} B^{3}(\log p),
$$

since $p^{1 / 2}<B \leqslant p$.

On the other hand $p \sum f_{B}(p x) \geqslant 0$ and, by part (iii) of Lemma 4,

$$
p^{-1} \sum_{\mathbf{x} \in \mathbb{Z}^{4}} f_{B}(\mathbf{x}) \geqslant(32 p)^{-1} \#\left\{\mathbf{x} \in \mathbb{Z}^{4} ;\left|x_{i}\right| \leqslant B / 4\right\} \gg p^{-1} B^{4} \text {. }
$$

If the implied constants in (8) and (9) are $c_{1}, c_{2}$ respectively, then Lemma 3 yields

$$
\sum_{\mathbf{x} \in \mathbb{Z}^{4}, p \mid Q(x)} f_{B}(\mathbf{x}) \geqslant c_{2} p^{-1} B^{4}-c_{1} p^{-1 / 2} B^{3}(\log p) \geqslant \frac{1}{2} c_{2} p^{-1} B^{4},
$$

providing that $B \geqslant 2 c_{1} c_{2}^{-1} p^{1 / 2}(\log p)$. Since the term $\mathbf{x}=\mathbf{0}$ contributes only $1=o\left(p^{-1} B^{4}\right)$ it follows from Lemma 4 part (i) that $p \mid Q(\mathbf{x})$ with some $\mathbf{x} \neq \mathbf{0}$ for which $\left|x_{i}\right| \leqslant$ $6 c_{1} c_{2}^{-1} p^{1 / 2}(\log p)$. This completes the proof of Theorem 1 . Note that it would not have been sufficient to use Lemma 5 in place of Lemma 6.

\section{REFERENCES}

1. Z. I. Borevich and I. R. Shafarevich, Number theory (Academic Press, New York, 1966).

2. G. H. Hardy and E. M. Wright, An introduction to the theory of numbers (Oxford University Press, 1960).

3. A. Schinzel, H.-P. Schlickewei and W. M. Schmidt, Small solutions of quadratic congruences and small fractional parts of quadratic forms, Acta Arith., 37 (1980), 241-248.

Magdalen College

OXFORD OX1 4AU 